International Journal of Wireless \& Mobile Networks (IJWMN) Vol. 4, No. 6, December 2012

\title{
DCT \& DWT IMAGES COMPRESSION ALGORITHMS IN Wireless SENSORS NETWORKS: COMPARATIVE STUdy AND PERFormanCE ANALYSIS
}

\author{
Oussama Ghorbel ${ }^{1}$ Walid Ayedi ${ }^{1}$ Mohamed Wasim Jmal ${ }^{1}$ and Mohamed Abid ${ }^{1}$ \\ ${ }^{1}$ National Engineers School of Sfax, Sfax University, Tunisia. CES Research Unit \\ oussama.ghorbel@gmail.com, ayediwalideieee.org , \\ wassim.jmal@gmail.com, mohamed.abid@enis.rnu.tn
}

\begin{abstract}
The recent availability of inexpensive hardware has enabled the new research field of wireless sensor networks. This is a network of interconnected devices, capable of retrieving images from the environment. The nodes, in this type of network, have very limited resources, in terms of processing unit, bandwidth and energy. Efficient coding of the image content is therefore important. In Wireless Sensors Network, many image compression algorithms are used. Indeed, the most popular methods such as JPEG or JPEG2000 can yield higher energy consumption than when transmitting uncompressed images. However, the most of the compression algorithms are inapplicable on sensor nodes because the limitation in terms of memory as well as processor speed.

To solve this problem, we use DWT and DCT image compression algorithm that allows an efficient tradeoff between energy consumption and image distortion. These experimental results demonstrated by the performance evaluation are in terms of image quality, execution time and memory space, as shown in this paper.
\end{abstract}

\section{KEYWORDS}

Wireless Sensors Networks (WSNs), Image compression, Discrete Wavelet Transform (DWT), Discrete Cosine Transform (DCT), Computational complexity, Energy consumption, Experimentation.

\section{INTRODUCTION}

Uncompressed data (graphics, audio and video) requires considerable storage capacity and transmission bandwidth. The demand for data storage capacity and data transmission bandwidth continues to outstrip the capabilities of available technologies, yet the rapid change in terms of the mass storage density, processor speed and system performance digital communication.

Previously, to capture data from the natural or built environment are not possible, but actually, with Wireless Sensor Networks (WSN), which is a new tool to sense all type of data, all is possible. In WSN, a tiny embedded device has the capability for sensing, processing and wireless communication.

Thanks to recent advances in microelectronics and wireless communications, it is predicted that wireless sensor networks (WSN) will become ubiquitous in our daily life and they have already been a hot research area for the past couple of years [1]. A wide range of emerging WSN applications, like object detection, surveillance, recognition, localization, and tracking, require vision capabilities [2].

Although, the hardware prerequisites are met, application aware and energy efficient algorithms for both the processing and communication of image have to be developed to make vision

DOI : 10.5121/ijwmn.2012.4604 
International Journal of Wireless \& Mobile Networks (IJWMN) Vol. 4, No. 6, December 2012

sensor applications feasible [3]. Most of the work in literature is devoted to image processing (data extraction, compression and analysis) (Tang and Raghavendra (2004); Magli et al. (2003); Song et al. (2006); Wagner et al. (2003)) while the case of image transmission over WSN is still in an earlier stage of investigation.

We propose in this paper, two image transmission scenario which both are based on discrete wavelet transform (DWT) and discrete cosine transforms (DCT) [4, 5] driven by energy efficiency considerations suitable for WSN.

In many applications based on image compression and transmission, intermediate nodes consuming much more energy to forwarding data toward the sink than nodes collecting and forwarding data such as temperature value. As the radio transceiver is one of the most power greedy components of sensor nodes, compression seems a natural answer to the image transmission problem over a WCSN. Unfortunately, most of the compression algorithms are inapplicable on sensor a node which is due to resource limitation in terms of memory or processor speed. For instance, Ferrigno et al. have shown in [6] that the energy consumption involved by popular algorithms such as JPEG, JPEG2000 or SPIHT could be even greater than for the uncompressed image case [7,8,9]. As a consequence, special challenges for energyefficient image compression in WSNs must be addressed to maintain a long network lifetime. These nodes are equipped with a processing unit with limited memory and energies resources and a communication unit, usually a radio transceiver. Nodes are powered by small batteries which generally cannot be changed. The application of multimedia (image, video, etc.) on wireless sensor networks is being, these days, a great requirement for the research and industrial community [10]. The current researches deal with image processing like data extraction, image processing and analysis [11]. Thus, the case of image compression and image transmission over WSN is not a new concept because there are many researchers who worked on it [12].

Our work is to experimentally test two techniques for image compression to ensure that they are effective or not for image transmission through wireless sensor network. The two selected techniques are from two different families which are: discrete wavelet transforms (DWT) and discrete cosine transforms (DCT). DWT is an operation that is applied on the entire image and allows image decomposition into separable subbands for multi-resolution representation purposes but DCT is applied on each block with size 8x8 and not on the entire image which allows a high compression ratio. The implementations of these methods on a real wireless sensor platform are allowed to reconcile with the real problems related to image processing applications. Wireless Sensor Network (WSN) [13] has been considered as an active area of research which many researcher worked on it and a wide range of applications have been developed. Sensor nodes are mainly characterized by their scarce resources and limited energy.

The rest of this paper is organized as follows: A state of the art, which deals with the various researches related to wireless sensors network, was presented in section 2. Section 3 and 4, gives an idea about compression methods and their advantages in general and in wireless sensor network. Section 5, it's a performance evaluation that describes the real implementation of DWT and DCT on TelosB sensor nodes. Section 6 and Section 7 present the applied scenario for the two compression technique DWT and DCT. A summary table between DWT and DCT is deals in Section 8. Section 9 shows quantity of consumed energy by the TelosB mote and how long time the network can be survived in section 10. Conclusion is drawn in section 11 .

\section{RELATED WORK}

Recently, WSN (Akylidiz et al. (2002)) has been an active area of research and a wide range of applications have been developed. Sensor nodes are mainly characterized by their scarce resources and limited energy. As a result, a considerable effort has been given to energy 
International Journal of Wireless \& Mobile Networks (IJWMN) Vol. 4, No. 6, December 2012

efficient data transmission schemes ranging from the hop by hop medium access control level (Ye and Heidemann (2003); Langendoen and Halkes (2005)) to that of sensor to sink data delivery (Heinzelman et al. (2000); Tian and Georganas (2003);Kim et al. (2004); Liu et al. (2004)).

Out of concern for energy efficiency, both image data processing and transmission must be considered. A lot of work has been done in data processing research area. Recent results on data compression and issues in their deployment in wireless sensor networks have been exposed by Tang and Raghavendra (2004) and Mishra et al. (2007). Chiasserini and Magli (2002); Gerla and $\mathrm{Xu}$ (2003); Magli et al. (2003) adopted JPEG with change/difference compression. This latter has the advantage of low processing power needs, however, it does not support error control.

In order to save energy, mainly in a densely deployed WSN, many proposals (Song et al. (2006); Wagner et al. (2003); Gerla and Xu (2003); Wu and Abouzeid (2004)) adopt a distributed coding approach where neighboring sensor nodes cooperate in performing image coding. However, a significant exchange of data, which would waste energy, may be necessary. Maniezzo et al. (2002) addressed the tradeoff between computing and communication and show that there are an optimal number of nodes involved in a distributed coding process which minimizes the total energy consumption. Wu and Abouzeid (2004) adopted a multi-layer coding using JPEG2000 based on wavelet compression. Two methods of parallelizing the compression process are proposed and compared in terms of consumed energy and image quality. Yu et al. (2004) used DWT in order to obtain different layers with different quality. There is a focus on point to point image transmission with energy saving considerations. The optimal number of layers to be transmitted and the optimal strategies for each layer are determined thanks to an algorithm that minimizes the overall processing and transmission energy consumption given the expected end-to-end distortion constraint. This algorithm has the following inputs: the estimated channel condition, the characteristics of image content, and the set of available coding and transmission strategies. Other approaches to save energy use buffering techniques (Magli et al. (2003); Wanghong and Nahrstedt (2003)). Another work proposed by Wanghong and Nahrstedt (2003)), they realized a scenario in which a source node buffers encoded frames and transmits them in bursts in order to better exploit idle intervals of the processor and network card. The most current research related to image compression in wireless sensor networks [14] are limited to the evaluation by simulation. The authors have used several methods in the compression CWHN as LBT, SPIHT [15], ISEC [16] tested by simulation and tested on a real platform on Mica2. For instance, ISEC makes a compression method at the source, which uses a coding block of $2 \times 2$ pixels and removes one pixel from the 4 to minimize the compression rate and then finds the missing pixel using three present pixels. In [17], there is a comparison between two models of selection zonal coefficient of the DCT, one using a square shaped area and the other a triangular area.

In our work, a considerable effort has been given to compare two models for image compression to ensure they are effective or not for image transmission through wireless sensor network called DWT and DCT. In our work, and compared to other projects in WSN, we focuses more on low-bitrate image transmission, image quality and execution time over long-range indoor and outdoor sensor networks.

The aim of our paper is as follow: Firstly, we compare two methods of compressing images which are the Discrete Cosine Transform (DCT) and Discrete Wavelet Transform (DWT) and test their capability on wireless sensor networks (WSNs). Secondly, implement these methods on a real WSNs platform with TelosB sensor type. Thirdly, we execute performance evaluation and we compare the two methods in terms of image quality, execution and transmission time, packet lost and memory usage and finally energy consumption. 
International Journal of Wireless \& Mobile Networks (IJWMN) Vol. 4, No. 6, December 2012

\section{IMAGE COMPRESSION}

\subsection{Preview}

To solve the problem of reducing the amount of data required to represent a digital image, we use Image compression method to remove the redundant data. Based on mathematical theory, we transforming a 2-D pixel array into a statistically uncorrelated data set which is applied prior to storage or to transmit the image. After some time, the compressed image is decompressed to reconstruct the original image. Image compression is recognized as a famous technology used until now to solve the compression problem. It plays a major role in many diverse applications, including remote sensing (weather and earth applications), medical imaging, facsimile transmission [18], video-conferencing, military and space application.

\subsection{Fundamentals of image compression}

To minimise a given quantity of information, we use a compression method which is refers to the process of reducing the amount of data. The neighboring pixels are correlated and therefore contain redundant information. So, the solution is to find less correlated representation of the image. There are two fundamental components of compression which are the redundancy and irrelevancy reduction: first, aims to removing duplication from the signal source and second, remove parts of the signal that will not be noticed by the signal receiver.

We show now a mathematically quantifiable entity but not an abstract concept. If $n 1$ and $n 2$ denote the number of information-carrying units in the two data sets that represent the same information, the relative data redundancy $\mathrm{RD}$ of the first data set (the one characterized by $\mathrm{n} 1$ ) can be defined as: $\mathrm{RD}=1-1 / \mathrm{CR}$, where $\mathrm{CR}$ called the compression ratio, so $\mathrm{CR}=\mathrm{n} 1 / \mathrm{n} 2$.

For the case when $\mathrm{n} 2=\mathrm{n} 1$ and $\mathrm{RD}=0$, the first representation of the information contains no redundant data. Then, if $\mathrm{n} 2<<\mathrm{n} 1$, so $\mathrm{CR}->\infty$ and $\mathrm{RD}->1$, implying significant compression and highly redundant data. Finally, in the case of $n 2>n 1, C R->0$ and RD $->-\infty$, we conclude that the second data set contains much more data than the original representation. Generally CR $=10(10: 1)$ defines that the first data set has 10 information carrying units for every 1 unit in the second or compressed data set. Thus, the redundancy of the data in the first data set is redundant with respect to the second one [19].

In order to be useful, a compression algorithm has a corresponding decompression algorithm that reproduces the original file once the compressed file is given. There are many types of compression algorithms developed.

\subsection{Image Compression and Reconstruction}

Three basic data redundancies can be categorized in the image compression standard.

- Spatial redundancy which is due to the correlation between neighboring pixels.

- Spectral redundancy due to correlation between the color components.

- Psycho-visual redundancy which is due to properties of the human visual system.

The spatial and spectral redundancies are present because certain spatial and spectral patterns between the pixels and the color components are common to each other, whereas the psychovisual redundancy originates from the fact that the human eye is insensitive to certain spatial frequencies. The principle of image compression algorithms are (i) reducing the redundancy in the image data and (or) (ii) producing a reconstructed image from the original image with the introduction of error that is insignificant to the intended applications. The aim here is to obtain an acceptable representation of digital image while preserving the essential information contained in that particular data set [20]. 


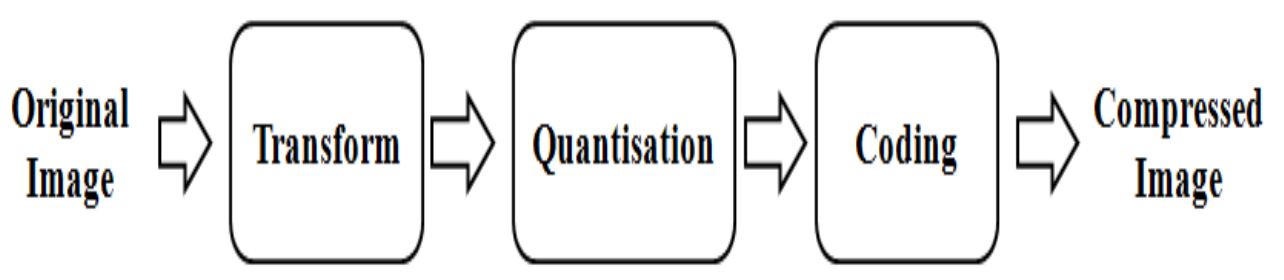

Figure 1. Image Compression System

The problem faced by image compression is very easy to define, as demonstrated in figure 1 . First the original digital image is usually transformed into another domain, where it is highly decorrelated by using some transform. This decorrelation concentrates the important image information into a more compact form.

The compressor then removes the redundancy in the transformed image and stores it into a compressed file or data stream. In the second stage, the quantisation block reduces the accuracy of the transformed output in accordance with some pre- established fidelity criterion. Also this stage reduces the psycho-visual redundancy of the input image. Quantisation operation is a reversible process and thus may be omitted when there is a need of error free or lossless compression. In last step of the data compression model the symbol coder creates a fixed or variable-length code to represent the quantizer output and maps the output in accordance with the code.

A variable length code is commonly used to represent the mapped and quantised data set. It assigns the shortest code words to the most frequently occurring output values and thus reduces coding redundancy. The operation in fact is a reversible one. The decompression reverses the compression process to produce the recovered image as shown in figure 2 . The recovered image may have lost some information due to the compression, and may have an error or distortion compared to the original image.

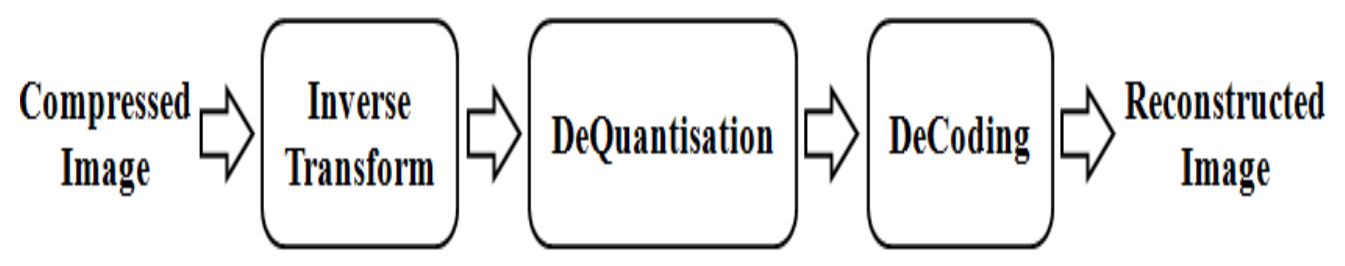

Figure 2. Image Decompression System

\section{COMPRESSION METHOD FOR SENSOR NETWORKS}

Compression method reduces the amount of data that the source will be transmitted. The data processing at the source is also necessary to anticipate the possible loss of information during the transmission of packets to the desired node, or to control the amount of data to send (the quality of the image) under the conditions of network such as the distribution of nodes and the distance separating them. There are many compression method used in literature for digital signal processing applications. We use in our work (in section IV) the two DWT and DCT transforms, and outline their relevance to the implementation of compression algorithm.

Data compression has several advantages such as:

- Extending the life of the source node. Unless the source has data to transmit, and consumes less energy at the radio transceiver. This is true as the complexity of the compression algorithm adopted is reliable enough to be profitable. The compression process should not cost more in terms of energy gain that it brings about 
International Journal of Wireless \& Mobile Networks (IJWMN) Vol. 4, No. 6, December 2012

communication, if the presence of a compression process could reduce the lifetime of the node.

- Extending the life of the intermediate nodes. For the same reasons, reducing the amount of data at the source will be necessarily beneficial to the nodes responsible for relaying packets between the source and the destination node. They receive fewer packets of data, so they have fewer packets and acknowledgments to transmit.

- Reduced risk of congestion. A decrease in the amount of data transmitted over the network will lead to reduced risk of network congestion, thus a decrease in packet loss and transmission delays.

- Tolerance to losses. Some reinforcement on tolerance to packet loss can be achieved by applying some processing mechanisms at the source, such as mixing or tattoo image.

\section{Performance evaluation}

We evaluate our work on the real platform named TelosB nodes. The comparison will be very detailed depending on the results obtained through the PSNR values taking into account studies on image quality. In our experiment, we evaluate two compression techniques in image processing applications. We focus on the following question: Are these techniques valid for the context of WSNs? For there, we follow the steps of scenarios, measurement parameters and materials used that allow us to do our assessment.

The performance of the two compression algorithm has been evaluated considering a JPEG compression chain that is including the quantization and Huffman tables. In the following, we discuss about performance of the two approaches regarding quality of the reconstructed images and energy consumption.

Experimental results in terms of quality are given in Figure 5 which represents image Lena by DCT [21] and DWT [22] for different values of k. Obviously, even if the overall visual quality is good, image distortion increases as $\mathrm{k}$ decreases for both proposed methods. Furthermore, DWT and DCT provide different level of quality (visually speaking and with respect to the PSNR) for large values of $k$ (i.e., 2 and 8 here). On the other hand, for small values of $k$ the DWT method offers a better performance, which is normal since more coefficients are processed.

To discuss energy consumption, we have considered the TelosB mote [23], an ultra-low power sensor plateform developed by the University of California, Berkeley. TelosB mote consists of a MSP430 16- bit microcontroller and a 802.15.4-compliant CC2420 radio transceiver. The microcontroller operates down to $1.8 \mathrm{~V}$ and consumes $3 \mathrm{~mW}$ in active mode (clock speed of 8 $\mathrm{MHz}$ ). The radio transceiver has a transmit power of $35 \mathrm{~mW}$ at $0 \mathrm{dBm}$ and provides a data rate of $250 \mathrm{kbps}$. We used also the TinyOS operating system to validate and measure the performance of our proposal. The TelosB motes have the following characteristics.

Tableau 1. Characteristics of Sensors Nodes

\begin{tabular}{|c|c|c|c|}
\hline Manufacturer & Processor & Program Memory & RAM \\
\hline Crossbow & IT MSP430 & $48 \mathrm{~KB}$ & $10 \mathrm{~KB}$ \\
\hline Clock & Radio Unit & Band/Data rate & Max Consumption \\
\hline $8 \mathrm{MHz}$ & $\begin{array}{c}\text { Chipcon } \\
\text { CC } 2420\end{array}$ & $2.4 \mathrm{GHz} / 250 \mathrm{kbps}$ & $1.8 \mathrm{~mA}$ \\
\hline
\end{tabular}

\subsection{The Process of the Algorithm Implementation using DCT}


International Journal of Wireless \& Mobile Networks (IJWMN) Vol. 4, No. 6, December 2012

Image compression at the sensor node includes several steps. First, image is transformed into a format suitable for image compression. Then, the image is split into 8x8 blocks. The next step involves transformation of the block into the frequency plane. This is done by using a fast discrete cosine transform (FDCT). To exploit spatial correlation between pixels, we use this transform. Most of information is concentrated to a few low-frequency components after the transformation.

To reduce the number of bits needed to represent the image, these components are then quantized. This step will lower the quality of the image by reducing the precision of the components. The trade-off between quality and produced bits can be controlled by the quantization matrix, which will define the step size for each of the frequency component. We use ZigZag method to put the most likely non-zero components first and the zero components in the bit-stream. We use then entropy coding which is a combination of variable length coding and Huffman encoding. Finally, we create a suitable data packet for transmission over the wireless sensor network.

\subsection{The Process of the Algorithm Implementation using DWT}

For the second algorithm, the image is transformed into a suitable format to an image compression. On the first level, the filters divide the input image into four non-overlapping multi-resolution sub-bands LL, LH, HL and HH. The sub-band LL represents the coarse-scale DWT coefficients while the sub-bands LH, HL and HH represent the fine-scale of DWT coefficients. We show that each subband contains the low-pass information and the others contain high-pass information of horizontal, vertical and diagonal orientation. The next step is quantification and coding of subbands used to reduce the number of bits needed to represent the image. This step will lower the quality of the image but not like DCT. The quality will depend on the value of the quantization used. The bit plane coding and subbands provides various coding modes, the compressed image can indeed be represented by increasing resolution or by increasing quality. The next step is arithmetic coding. It is a variable length coding. Unlike other encodings, it encodes the source message fully and represents a single number. Finally, we create a suitable data packet for transmission over the wireless sensor network.

\section{APPLIED SCENARIO FOR THE TWO COMPRESSION TECHNIQUES DWT AND DCT}

\subsection{First Scenario}

In this scenario, we have used 2 TelosB motes. Compression and decompression are performed respectively at the source and destination nodes. For radio communication, our implementation is based on 802.15.4 PHY and MAC layers which is a standard protocol for low-rate wireless personal area networks (LR-WPANs). 


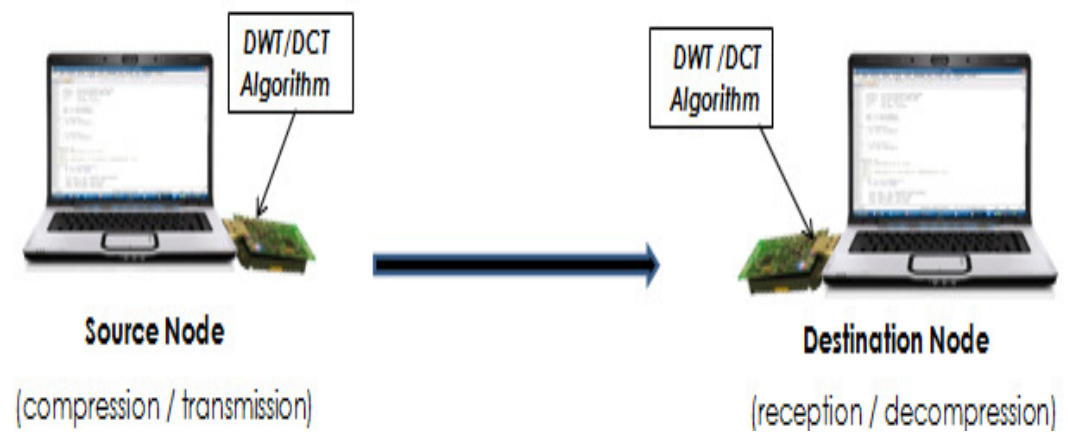

Figure 3. From the source to destination nodes using radio communication

There are different images uses for experimentation, we choose for example Lena to do the different scenario.

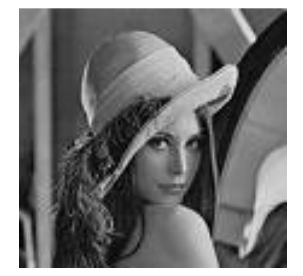

Figure 4. Original Image

\subsection{Peak Signal to Noise Ratio (PSNR)}

As a measure of the quality of image, the peak signal to noise ratio (PSNR) is typically used. This PSNR ratio expresses the difference in quality among the original Lena image and the decomposed one, while the higher the PSNR is, the better the quality of the decomposed image is. The expression of PSNR in decibels $(\mathrm{dB})$ is given below in (1).

$$
\begin{aligned}
& P S N R=10 \log _{10}\left(\frac{255^{2}}{M S E}\right) \\
& \text { where: } \quad M S E=\frac{1}{m n} \sum_{i=0}^{m-1} \sum_{j=0}^{n-1}\left\|I_{0}(i, j)-I_{r}(i, j)\right\|^{2} \\
& m, n=\text { image size }>0, \\
& I_{0}=\text { original value, } \\
& I_{r}=\text { compressed value }
\end{aligned}
$$

The following figure illustrates the obtained PSNR by varying nodes distance for $64 \times 64$ images. According to these figures, images quality is better using DWT regardless of the 
International Journal of Wireless \& Mobile Networks (IJWMN) Vol. 4, No. 6, December 2012

distance and resolution. For distances higher than $30 \mathrm{~m}$, a higher gap of PSNR values is obtained. Quality is reduced when increasing distances between source and destination nodes.

The reconstructed images (Lena) for different distance (d) between sensor nodes are given by figure 5 .

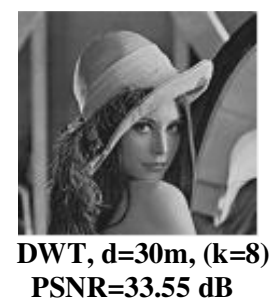

PSNR=33.55 dB

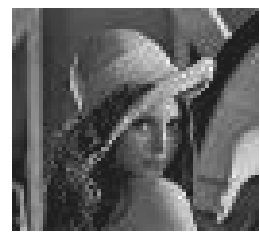

DCT, $d=30 \mathrm{~m},(\mathrm{k}=8)$ PSNR=30.52 dB

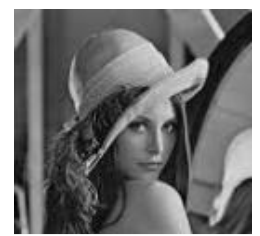

DWT, $d=30 \mathrm{~m},(\mathrm{k}=2)$ PSNR=32.47 dB

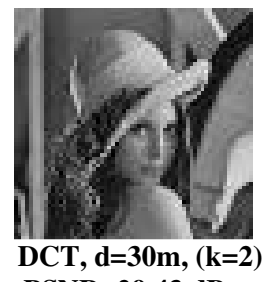

PSNR=29.43 dB

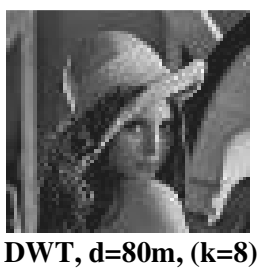

PSNR $=26.47 \mathrm{~dB}$

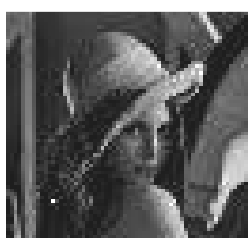

DCT, $d=80 \mathrm{~m},(\mathrm{k}=8)$ PSNR=24.35 dB

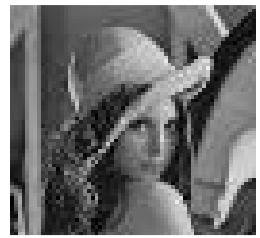

DWT, $d=80 \mathrm{~m},(\mathrm{k}=2)$ PSNR $=25.58 \mathrm{~dB}$

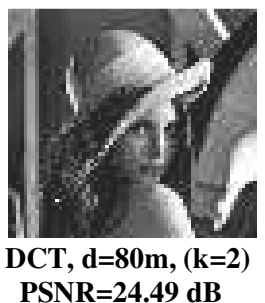

Figure 5. Obtained images with different distance for Lena $64 \times 64$

We conclude that the image quality decreases when the distance between the nodes increases. We show that at some distances we get blurred images that are due to packet loss. In addition, the image size has a great influence. A question can be asked that why we used the Lena image with only two sizes $32 \times 32$ and $64 \times 64$ and not other sizes? The answer is simple because it depend by the memory available by the sensors and consequently it affects the transmission time because the coding phase depend of the microcontroller used (hardware). We can of course use images size $128 \times 128$ and more, but the coding phase will be processed by the $\mathrm{PC}$ which it won us in terms of transmission time which is almost equal to $50 \%$. 
International Journal of Wireless \& Mobile Networks (IJWMN) Vol. 4, No. 6, December 2012

\section{TranSMISSION WiTH AN INTERMEDIATE NODE}

\subsection{Second Scenario}

We are interested in this section to a topology with larger number of nodes. Our experiments were performed on TelosB wireless sensor nodes.

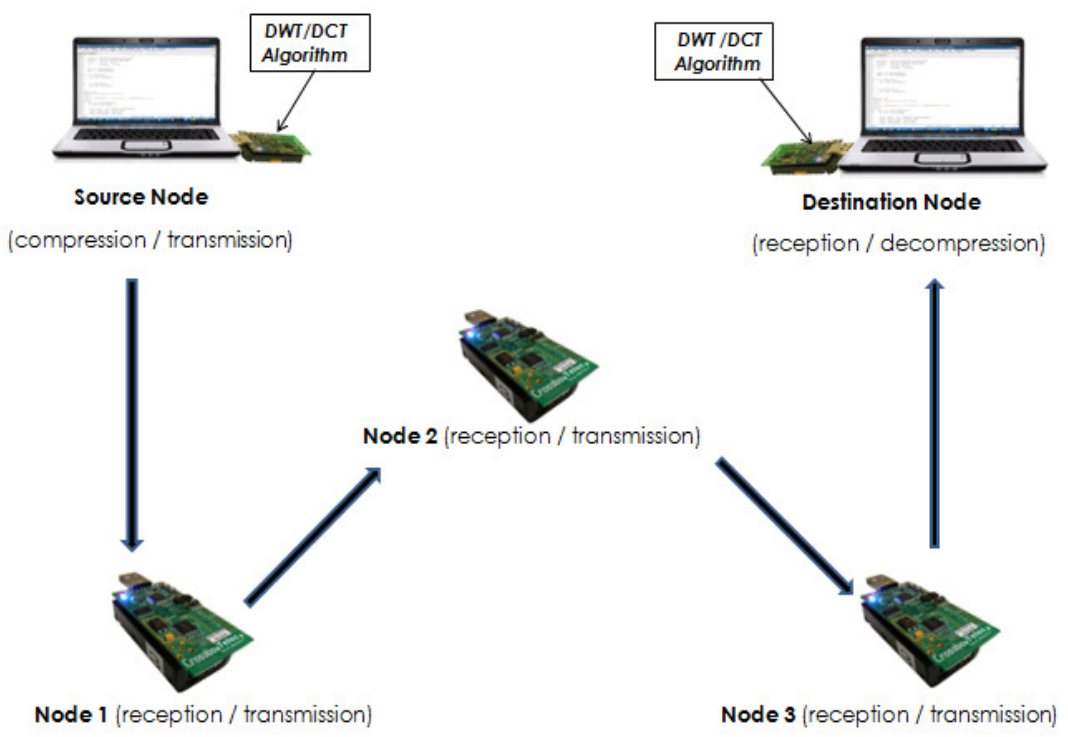

Figure 6. Transmission scenario with intermediate nodes.

This figure represents an image transmission scenario from source to destination node through intermediate node 1 , node 2 and node 3 . Compression and decompression are performed respectively at the source and destination nodes. Intermediate nodes only transmit compressed images. We used different image types and different resolution. We varied distance between source and destination nodes. For each configuration, tests are performed with DCT and DWT. Measured parameters are the quality of reconstructed images (using PSNR).

\subsection{Obtained Images with an Intermediate Node}

This figure shows the reconstructed images obtained with the use of intermediate sensors that are used across different distances. The first two images represent the images using DCT and the last two images are obtained by using the DWT technology.

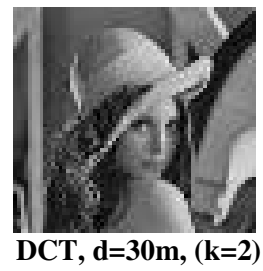

PSNR=28.54 dB

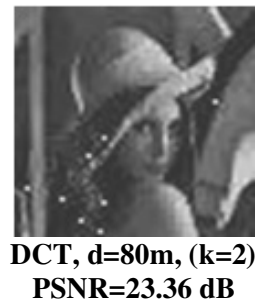

PSNR=23.36 dB 
International Journal of Wireless \& Mobile Networks (IJWMN) Vol. 4, No. 6, December 2012
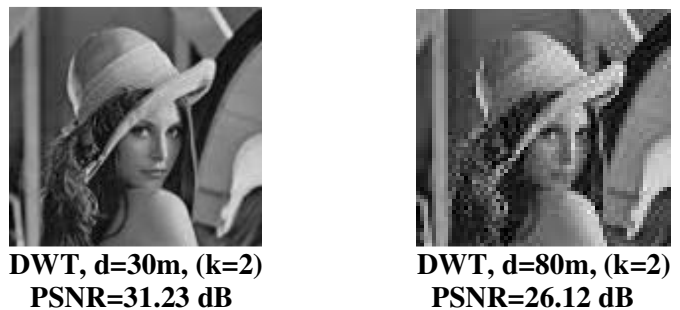

Figure 7. Obtained images with different distance for Lena $64 \times 64$

From this figure, we can conclude that the more we increase the distance, the more there is degradation. Thus, it is clear that there is a difference between the images using DCT and DWT. In fact, the compression based on DWT gave better results in terms of image quality and energy consumption.

\section{SUMMARY TABLE: DWT VS DCT}

Table 2 shows a comparison between DWT and DCT with the evaluation criteria terms: Compression Performance, Execution Time and used Memory. Character $(+)$ refers the quality and performance task. When the number of $(+)$ increases, it means that the operation is successfuly used by this algorithm and gives good results.

Tableau 2. Comparative criterion between DWT and DCT

\begin{tabular}{|c|c|c|}
\hline $\begin{array}{c}\text { Efficiency } \\
\text { Criterion }\end{array}$ & $D C T$ & $D W T$ \\
\hline $\begin{array}{l}\text { Compression } \\
\text { performance }\end{array}$ & +++ & +++++ \\
\hline Execution Time & + & +++ \\
\hline Memory Used & +++ & + \\
\hline
\end{tabular}

We finally note from this table that the DWT is better than the DCT in terms of compression performance and execution Time in the context of Wireless Sensors Networks using TelosB motes.

\section{Energy Consumption Measurement}

The experiment of measuring energy consumption in the WSN show that the average voltage corresponding to the maximum energy consumption is approximately: V Havg $=212.9$ $\mathrm{mV}$. This value assumes that the microcontroller and radio transceiver are you running (ie 
International Journal of Wireless \& Mobile Networks (IJWMN) Vol. 4, No. 6, December 2012

processing and radio transmission in progress). The intensity when the radio transceiver of the sensor Telosb we used in our experience, is off is not zero, the consumption of the microcontroller must be considered and it is: I Lavg $=1800 \mathrm{uA}$.

Considering both values and the measured value of the resistance is $10 \mathrm{ohms}$ connected in series, it is possible to calculate the batteries lifetime.

\section{BATTERIES LIFETIME}

The most suitable power source for the motes is batteries. Each mote needs $3 \mathrm{~V}$ to work, or, considering batteries, two units of AA batteries.

Nowadays, market is full of different brands that offer many different kinds of batteries, but, considering the low environmental impact of the network, the most suitable are rechargeable batteries. The reason to choose this kind of batteries is they can be recharged several times, which involves saving money in batteries and the possible contamination that batteries can cause if they are not recycled in the correct way.

Rechargeable batteries can be based on different technologies. The first one where based on $\mathrm{NiCd}$, but due to memory effect, after each recharge the life cycle was reduced. Now the principal technology is NiMH; they have no memory effect and higher charge and discharge capacity which makes them more useful than the previous ones.

One of the possible rechargeable batteries to be used is Alkaline AA-NiMH $2850 \mathrm{mAh}$.

Tableau 3. Battery characteristics

\begin{tabular}{|l|l|}
\hline Maximum charge voltage & $1.5 \mathrm{~V}$ \\
\hline Nominal voltage & $1.2 \mathrm{~V}$ \\
\hline Nominal capacity & $2850 \mathrm{mAh}$ \\
\hline Standard charge (current charge /charge time) & $270 \mathrm{~mA} / 16 \mathrm{~h}$ \\
\hline Fast Charge (current charge /charge time) & $2700 \mathrm{~mA} / 1.1 \mathrm{~h}$ \\
\hline
\end{tabular}

We then calculated the duration of battery life (estimated theoretical). According to the experiment, the intensity of the average load of the battery is given by equation (2) follows:

$$
I_{\text {Rag }}=\left(V_{\text {Hag }} / R^{*} \underline{T H}+I_{\text {Lag }} * \underline{T} \underline{L} /(\underline{T} H+\underline{T} L)\right.
$$

V Havg was measured over $\mathrm{R}=10 \Omega$ and I Pavg $=11,623 \mathrm{~mA}$ Max.

Considering the nominal capacity shown in previous table, it is obtained:

$$
\frac{\text { Capacity }}{I_{\text {Pavg }}}=\frac{2850 \mathrm{mAh}}{11.623 \mathrm{mAh}}=245.20 \mathrm{~h}
$$


International Journal of Wireless \& Mobile Networks (IJWMN) Vol. 4, No. 6, December 2012

Total DCT time is $245.20 \mathrm{~h}=10$ days, 5 hours, 20 minutes

Total DWT time is $281.34 \mathrm{~h}=11$ days, 17 hours, 34 minutes

Those values represent the total of energy consumption and battery life time. So, there is no big difference between de duration of time of DWT and DCT, but the method using DWT is more adopted in our experimentation because it gives a perfect performance in terms of image quality and energy consumption.

\section{Conclusions}

In this paper we have proposed and evaluated two image compression methods dedicated to transmission over Wireless Sensor Networks (WSNs) in order to decrease the energy consumption of sensors and thus to maintain a long network lifetime.

Despite discrete cosine transforms is considered as a good compression algorithm that gives a high compression ratio, the discrete wavelet transform have been widely recognized to be more prevalent than others. Due to the wavelets' excellent spatial localization, frequency spread, and multi-resolution characteristics, this algorithm is considered similar to the theoretical models of the human visual system. In future, our works will be concerned by transposing these results to other fast implementation methods of the literature and to other real sensor platforms. In addition, we aim to implement our proposal on dedicated platform for multimedia wireless sensor networks (MWSNs).

\section{REFERENCES}

[1] Naumowicz, Freeman, Heil, Calsyn, Hellmich, Brandle, Guilford \& Schiller (2008). "Autonomous monitoring of vulnerable habitats using a wireless sensor network". In : Proceedings of the Workshop on Real-World Wireless Sensor Networks, REALWSN'08. Glasgow, Scotland.

[2] Marcelloni \& Vecchio (2009). “An Efficient Lossless Compression Algorithm for Tiny Nodes of Monitoring Wireless Sensor Networks”. The Computer Journal 52(8), pp 969-987.

[3] Akyildiz, Melodia, Kaushik \& Chowdhury (2007). "A survey on wireless multimedia sensor networks". Journal Computer Networks: The International Journal of Computer and Telecommunications Networking, Volume 51, Issue 4, Inc . New York, NY, USA, United State.

[4] Liang and Tran (2001). Fast Multiplierless Approximations of the DCT with the Lifting Scheme. IEEE Trans. on Signal Processing, 49(12): 3032-3044.

[5] Jeong, Kim and Cho (2004). Low-Power Multiplierless DCT Architecture Using Image Correlation. IEEE Trans. on Consumer Electronics, 50(1): 262-267.

[6] Ferrigno, Marano, Paciello \& Pietrosanto (2005). "Balancing computational and transmission power consumption in wireless image sensor networks". In: IEEE International Conference on Virtual Environments, Human-Computer Interfaces, and Measures Systems (VECIMS 2005). Giardini Naxos, Italy.

[7] Mammeri, Khoumsi, Ziou and Hadjou (2008). "Modeling and Adapting JPEG to the Energy Requirements of VSN". In International Conference on Computer Communications and Networks (ICCCN 2008), Proceedings, 806-811, St. Thomas, Virgin Islands-USA.

[8] Feig and Winograd. Fast algorithms for the discrete cosine transform. IEEE Trans. on Signal Processing, 40(9): 2174-2193, 1992.

[9] C. N. Taylor and S. Dey. Adaptive Image Compression for Wireless Multimedia Communication. In IEEE International Conference on Communications (ICC 2001), Proceedings, 6: 1925-1929, Helsinki-Finland, June 2001. 
International Journal of Wireless \& Mobile Networks (IJWMN) Vol. 4, No. 6, December 2012

[10] Enesi, Zanaj, Kamo, Kolici, Shurd (2010). "Image Compression for Wireless Outdoor Sensor Networks". BALWOIS 2010 - Ohrid, Republic of Macedonia.

[11] Soro \& Heinzelman (2009). "A survey of visual sensor networks. Advances in Multimedia": Article ID 640386, pp 21.

[12] Tang \& Raghavendra (2004). "Compression techniques for wireless sensor networks". Wireless Sensor Networks, chapter Kluwer Academic Publishers 2004.

[13] Akyildiz, Melodia \& Chowdhury (2007). "Wireless multimedia sensor networks: a survey". IEEE Wireless Communications Magazine, 32-39.

[14] Chew, Ang, \& Seng (2008), "Survey of Image Compression Algorithms in Wireless Sensor Networks”, in Proc. of Int. Symp. on Information Technology (ITSim 2008).

[15] Li, Peng Pang \& Liu (2010). "SPIHT algorithm combined with Huffman encoding”. Third International Symposium on Intelligent Information Technology and Security Informatics.

[16] Duran-Faundez (2009). “Transmission d'images sur les réseaux de capteurs sans fils sous la contrainte de l'énergie". Thesis, Nancy University.

[17] Makkaoui, Lecuire, \& .Moureaux (2010). "Fast Zonal DCT-based image compression for Wireless Camera Sensor Networks", in "2nd International Conference on Image Processing Theory, Tools and Applications, IPTA 2010, Paris : France (2010)" DOI : 10.1109/IPTA.2010.5586798.

[18] Digital Compression and coding of Continuous-tone still images, part 1, requirements and Guidelines. ISO/IEC JTC1 Draft International Standard 10918-1, Nov. 1991.

[19] Gonzalez and Woods, "Digital Image Processing”, Reading. MA: Addison Wesley, 2004.

[20] David Salomon, "Data Compression". The Complete Reference, 2nd Edition Springer-Verlag 1998.

[21] Caben \& Gent (2008). "Image Compression and the Discret Cosine Transform, in International Conference on Machine Learning and Cybernetics, pp. 407- 410.

[22] Antonini, Barlaud, Mathieu \& Daubechies (1992). "Image coding using wavelet transform". IEEE Transactions on Image Processing 1, 2, 205-220.

[23] Crossbow, Telosb datasheet, www.xbow.com, Document part Number: 6020-0094-01 Rev B.

\section{Authors}

Oussama GHORBEL received the MS Degree in New Technologies of Dedicated Computer Science Systems from National Engineering School of Sfax, Tunisia in 2010. He is a Ph.D student at the National Engineering School of Sfax since January 2011. He is an invited PhD student in University of Technology of Troyes, France, at the Laboratory of Systems Modelling and Dependability. His research activity is conducted within CES research unit. He has received the Diploma degree in Computer Science, from the Faculty of Sciences of Sfax, Tunisia in 2007, the Engineering degree from the National Engineering School of

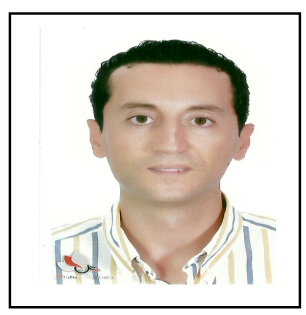
Sfax, in 2009. His current research interests are in the field of Wireless Sensor Networks (WSN) and Image Compression. He served in national and international conference organization: ICM, TWESD, Sensor-Nets.

Contact Information:

BP 1173, Sfax 3000, Tunisia

Email: oussama.ghorbel@gmail.com 
Walid AYEDI received the MS Degree from National Engineering School of Sfax, Tunisia in 2008. He is a PhD student at Computer \& Embedded Systems Laboratory, Tunisia. He is actually an invited $\mathrm{PhD}$ student in University of Technology of Troyes, France, at the Laboratory of Systems Modelling and Dependability. His research interests include image analysis, computer vision and machine learning. He served in national and international conference organization: IDT, ICM, TWESD, SensorNets.

Contact Information:

BP 1173, Sfax 3000, Tunisia

Email: ayediwalid@ieee.org

Mohamed Wassim JMAL is a Ph.D student at the National Engineering School of Sfax, Tunisia since 2008. His research activity is conducted within CES Laboratory. He has received the Engineering degree in Electrical Engineering, from the National Engineering School of Sfax in 2005 and the Master degree in Automatic and Industrial Informatics, from the same Engineering School, in 2007. His current research interests are in the field of Wireless Sensor Networks (WSN) and the Embedded Systems. They are focused on the implementation of wireless sensor networks applications in Reconfigurable System

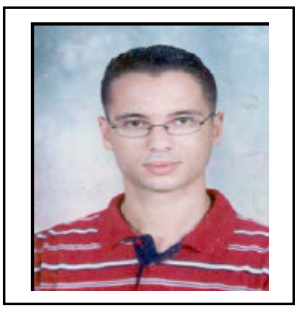
(http://www.ceslab.org/fr/perso.php?id=61). He has several publications in many conferences and Journals. He is working now as Assistant in Higher Institute of Applied Science and Technology of Gafsa, Tunisia. Mohamed Wassim JMAL served in national and international conference organization: IDT, ICM, TWESD, SensorNets.

Contact Information:

BP 1099, Sfax 3000, Tunisia

Tel : (+216) 22917723 - (+216) 74450775

Email: mohamed.wassim-jmal@ ceslab.org

Mohamed ABID, Head of «Computer Embedded System » laboratory CESENIS, Tunisia. Mohamed ABID is working now as a Professor at the Engineering National School of Sfax (ENIS), University of Sfax, Tunisia (http:// www. ceslab. org/ eng/ perso.php id =27). He received the Ph. D. degree from the National Institute of Applied Sciences, Toulouse (France) in 1989 and the "thèse d'état" degree from the National School of Engineering of Tunis (Tunisia) in 2000 in the area of Computer Engineering \& Microelectronics. His current research interests include: hardware-software co-design, System on Chip, Reconfigurable System, and Embedded System, etc. He has also been

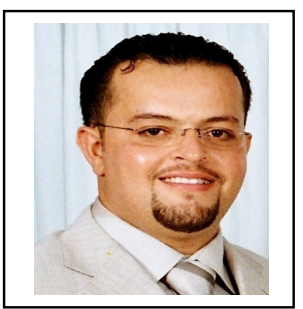
investigating the design and implementation issues of FPGA embedded systems. Actually Dr. Abid occupies the post of director of doctoral school "Sciences \& Technologies", University of Sfax. He is founding member and Head of the research laboratory «Computer Embedded System »CES-ENIS, since 2006 (http :// www. ceslab.org). He was Founding member of "System on Chip at Computer, Electronic and Smart engineering system” Laboratory at ENIS 2001-2005.

Contact Information:

BP 1173, Sfax 3000, Tunisia

Email: mohamed.abid@enis.rnu.tn 